\title{
BMJ Open The effect of blue-blocking intraocular lenses on circadian biological rhythm: protocol for a randomised controlled trial (CLOCK-IOL colour study)
}

Tomo Nishi, ${ }^{1}$ Keigo Saeki, ${ }^{2}$ Kenji Obayashi, ${ }^{2}$ Kimie Miyata, ${ }^{1}$ Nobuhiro Tone, ${ }^{3}$ Hiroki Tsujinaka, ${ }^{1}$ Mariko Yamashita, ${ }^{1}$ Naonori Masuda, ${ }^{1}$ Yutarou Mizusawa, ${ }^{1}$ Masahiro Okamoto, ${ }^{1}$ Taiji Hasegawa, ${ }^{1}$ Shinji Maruoka, ${ }^{1}$ Tetsuo Ueda, ${ }^{1}$ Masashi Kojima, ${ }^{1}$ Toyoaki Matsuura, ${ }^{1}$ Norio Kurumatani, ${ }^{2}$ Nahoko Ogata ${ }^{1}$

To cite: Nishi T, Saeki $K$, Obayashi K, et al. The effect of blue-blocking intraocular lenses on circadian biological rhythm: protocol for a randomised controlled trial (CLOCK-IOL colour study). BMJ Open 2015;5:e007930. doi:10.1136/bmjopen-2015007930

- Prepublication history for this paper is available online. To view these files please visit the journal online (http://dx.doi.org/10.1136/ bmjopen-2015-007930).

Received 11 February 2015 Revised 16 April 2015 Accepted 20 April 2015

\section{CrossMark}

${ }^{1}$ Department of

Ophthalmology, Nara Medical University School of

Medicine, Nara, Japan

${ }^{2}$ Department of Community Health and Epidemiology, Nara Medical University School of Medicine, Nara, Japan

${ }^{3}$ Center for Academic Industrial and Governmental Relations, Nara Medical University School of Medicine, Nara, Japan

Correspondence to Dr Keigo Saeki; saekik@naramed-u.ac.jp

\section{ABSTRACT}

Introduction: Blue light information plays an important role in synchronising internal biological rhythm within the external environment. Circadian misalignment is associated with the increased risk of sleep disturbance, obesity, diabetes mellitus, depression, ischaemic heart disease, stroke and cancer. Meanwhile, blue light causes photochemical damage to the retina, and may be associated with agerelated macular degeneration (AMD). At present, clear intraocular lenses (IOLs) and blue-blocking IOLs are both widely used for cataract surgery; there is currently a lack of randomised controlled trials to determine whether clear or blue-blocking IOLs should be used.

Methods and analysis: This randomised controlled trial will recruit 1000 cataract patients and randomly allocate them to receive clear IOLs or blue-blocking IOLs in a ratio of 1:1. The primary outcomes are mortality and the incidence of cardiovascular disease, cancer and AMD. Secondary outcomes are fasting plasma glucose, triglycerides, cholesterol, glycated haemoglobin, sleep quality, daytime sleepiness depressive symptoms, light sensitivity, the circadian rhythm of physical activity, wrist skin temperature and urinary melatonin metabolite. Primary outcomes will be followed until 20 years after surgery, and secondary outcomes will be assessed at baseline and 1 year after surgery.

Ethics and dissemination: Ethical approval has been obtained from the Institutional Review Board of Nara Medical University (№. 13-032). The findings of this study will be communicated to healthcare professionals, participants and the public through peer-reviewed publications, scientific conferences and the University Hospital Medical Information Network Clinical Trials Registry (UMIN-CTR) home page.

Trial registration number: UMIN000014680.

\section{INTRODUCTION}

Circadian misalignment between the internal biological rhythm and the external

\section{Strengths and limitations of this study}

- This is the first randomised controlled trial comparing the effect of blue-blocking intraocular lens for cataract surgery on mortality, the incidence of cardiovascular disease, stroke, cancer and age-related macular degeneration.

- To clarify the mechanism of the effects, we simultaneously measure circadian rhythm parameters such as urinary melatonin metabolite, wrist skin temperature and circadian physical activity rhythm.

- A limitation of this study is the lack of information about light exposure at night.

environment, such as behaviour and light exposure cycle, results in a number of negative health consequences. Epidemiological studies among night shift workers have shown significant associations between circadian misalignment and systemic diseases such as sleep disturbance, ${ }^{1}$ hip fracture, ${ }^{2}$ obesity and dyslipidemia, ${ }^{3}{ }^{4}$ diabetes mellitus, ${ }^{5}{ }^{6}$ depression, ${ }^{7}$ ischaemic heart disease and stroke ${ }^{8-10}$ and cancer. ${ }^{1-14}$ The mechanism is partly explained by decreased melatonin among shift workers ${ }^{15}$ and the findings from experimental studies in controlled laboratory conditions showing that circadian misalignment induced by a $28 \mathrm{~h}$ sleep-wake cycle under dim light conditions increased glucose, insulin and blood pressure, and decreased leptin and sleep efficiency. ${ }^{16}$

Light is the most important cue of the circadian biological rhythm, because it regulates the timing of the internal biological rhythm according to the phase response curve to light. ${ }^{17}$ Amplitude change due to light exposure was also reported from mammalian cell culture. ${ }^{18}$ Non-visual light information, a specific signal for the circadian 
system, perceived by intrinsically photosensitive retinal ganglion cells (ipRGCs) that contain melanopsin, is transmitted to the master circadian oscillator located in the suprachiasmatic nucleus via the retinohypothalamic tract. ${ }^{19}$ The action spectrum of light information for the circadian biological rhythm shows a peak at a shorter wavelength $(464 \mathrm{~nm})$ than that for visual information (approximately $555 \mathrm{~nm}$ ). ${ }^{20}$

According to the $\mathrm{WHO}$, cataract is a leading cause of blindness $(51 \% \text { of cases worldwide })^{21}$ and a common eye condition in elderly individuals. Opacity of the lens due to cataract reduces the transmission of light, especially the shorter wavelengths, ${ }^{22}$ and may cause circadian misalignment and its related diseases. In fact, according to observational studies, cataract surgery to replace clouded lenses with artificial intraocular lenses (IOLs), which increase light transmission, may improve not only visual acuity and quality of life ${ }^{23}$ but also sleep quality, ${ }^{24-27}$ depressive moods ${ }^{28-31}$ and life expectancy. ${ }^{29}$ The effectiveness of bright light therapy to decrease depressive symptoms ${ }^{32}$ and increase sleep quality ${ }^{33}$ supports the mechanism of the improvement of circadian rhythm alignment after cataract surgery.

For cataract surgery, clear IOLs that block ultraviolet (UV) B $(<320 \mathrm{~nm})$ and UVA $(320-400 \mathrm{~nm})$ radiation have been used since the early 1880s. In the 1990s, blue-blocking IOLs were introduced to prevent retinal phototoxicity caused by shorter wavelength light. Blue-blocking IOLs reduce transmission of light at $460 \mathrm{~nm}$ by $64-77 \%$ compared with clear IOLs which allow $>95 \%$ transmission of light at this wavelength. ${ }^{34}$ As the circadian timing system is most sensitive to light at $460 \mathrm{~nm}$, several reviews have raised the possibility that the use of blue-blocking IOLs could reduce the benefit of cataract surgery in terms of the circadian biological rhythm. ${ }^{35} 36$

Experimental studies have shown that blue light causes photochemical damage to the retinal pigment epithelium (RPE) in the presence of lipofuscin, which accumulates with ageing. Fluorophore A2E, a major component of RPE lipofuscin, mediates cell apoptosis under blue light radiation but not under green light radiation. ${ }^{37-39}$ Several cohort studies have shown that patients undergoing cataract surgery have an increased risk of age-related macular degeneration (AMD) during follow-up. ${ }^{40-43}$ A dramatic increase in transmitted blue light radiation through artificial IOLs after cataract surgery may partly explain the increased risk of AMD. In an in vitro study of RPE cell cultures under blue light radiation, blueblocking IOLs that were developed to protect against AMD decreased cell death by approximately $50 \% .{ }^{44}$

Although previous research has suggested benefits and disadvantages of blue-blocking IOLs, clear and blueblocking IOLs are both widely used for cataract surgery at present. It yet remains to be clarified whether clear or blue-blocking IOLs should be used for routine cataract surgery. The purpose of the present randomised controlled study is to compare all-cause mortality, the incidence of cardiovascular disease (CVD) and the incidence of cancer associated with circadian rhythm misalignment between participants after cataract surgery with implantation of clear or blue-blocking IOLs, and to determine whether blue-blocking IOLs reduce the incidence of AMD after cataract surgery compared with clear IOLs.

The CLOCK-IOL colour (Cataract Surgery and Circadian Biological Rhythm among Japanese Older People with Cataract in Nara, Kansai Region: Influence of Intraocular Lens Implantation) study is a parallel group, open label, randomised controlled study. After baseline assessment, all participants will be allocated to receive either clear IOLs (clear IOL group) or blueblocking IOLs (blue-blocking IOL group) in a 1:1 ratio. The outcomes among both groups will be followed at 1 year intervals.

\section{MATERIALS AND METHODS \\ Participants}

All procedures will be conducted at the Nara Medical University Hospital in Japan.

Ophthalmologists will assess the eligibility of patients diagnosed as having cataracts in Nara Medical University Hospital for the present study according to the following inclusion and exclusion criteria.

Inclusion criteria:

- Patients scheduled for the first cataract surgery

- Age $\geq 60$ years

- Cataract with grade $\geq 2$ nuclear opacification according to Lens Opacities Classification System III. ${ }^{45}$

Exclusion criteria:

- Severe mental illness or dementia

- Severe corneal opacities with difficulty in assessment of lens opacity or fundal examination

- Glaucoma with a visual field deficit with least mean deviation $>14 \mathrm{~dB}$ (Humphrey perimeter)

- Vitreous haemorrhage

- Proliferative diabetic retinopathy

- Macular oedema

- AMD

- Patients needing immediate cataract surgery

- Patients needing combined cataract and glaucoma surgery or combined cataract surgery and vitrectomy.

Participants requiring cataract surgery for one eye or both eyes will be included. For participants requiring surgery in both eyes, the intervention will be completed within 1-2 weeks, and the same type of IOL will be used in both eyes.

\section{Intervention}

The intervention in the present study is phacoemulsification with a small incision and implantation of an IOL. Participants will be randomly allocated to the clear or blue-blocking IOL group in a ratio of 1:1. In the clear IOL group, a clear spherical IOL (SA60AT, Alcon, Fort Worth, USA) will be implanted. In the blue-blocking 
IOL group, a spherical blue-blocking IOL (SN60AT) or an aspherical blue-blocking IOL (SN60WF, Alcon, Fort Worth, USA) will be implanted in a randomly allocated 1:1 ratio. Blue-blocking lenses with $+20.0 \mathrm{D}$ transmit $34 \%, 47 \%$ and $64 \%$ of light at 420,440 and $460 \mathrm{~nm}$, respectively. ${ }^{34}$ Before cataract surgery, the axial length of the eye will be measured with an A-scan UD-6000 (Tomey, Nagoya, Japan). The appropriate power of each IOL will be estimated using the SRK/T formula. ${ }^{46}$

\section{Primary outcomes}

The primary outcomes of the present study are mortality and the incidence of CVD, cancer and AMD after surgery.

\section{Secondary outcomes}

The secondary outcomes are listed below:

- Glucose/lipid metabolism indicators including glycated haemoglobin (HbAlc), fasting plasma glucose (FPG), triglyceride (TG), low-density lipoprotein cholesterol (LDL-C) and high-density lipoprotein cholesterol (HDL-C)

- Obesity as determined by body mass index and abdominal circumference

- Indicators of circadian rhythm, including urinary melatonin metabolite (6-sulfatoxymelatonin (aMT6-s)), wrist skin temperature and the circadian rhythm of physical activity

- Sleep quality based on actigraphic sleep quality, the Pittsburgh Sleep Quality Index (PSQI) and the Epworth Sleepiness Scale (ESS)

- The presence of depressive symptoms assessed using the short version of the Geriatric Depression Scale (GDS-15)

- Light sensitivity assessed by the post-illumination pupil response (PIPR)

- Ophthalmic parameters including visual acuity, the amplitude of pseudoaccommodation, the thickness of the retina and choroid measured using spectraldomain optical coherence tomography (SD-OCT), density of the macular pigment, aberration and subjective visual function assessed using the National Eye Institute Visual Function Questionnaire (NEI VFQ25).

\section{Participant timeline}

After baseline assessment and the surgical intervention, all participants will be requested to visit Nara Medical University Hospital annually (table 1). All outcomes will be assessed at baseline and 1 year after surgery. At annual follow-up visits from 2 to 20 years after surgery, details of mortality, the incidence of CVD, cancer and AMD will be recorded; the questionnaire survey will be administered; SD-OCT will be performed and density of the macular pigment will be measured.

\section{Mortality and disease incidence}

The incidence of CVD and cancer will be assessed using a self-administered questionnaire, and the diagnosis will be confirmed based on medical records. When a participant dies, the cause of death will be determined from the medical records and the death certificate. The presence of AMD at each visit will be investigated by fundus examination and SD-OCT (Spectralis; Heidelberg Engineering, Heidelberg, Germany).

\section{Ocular examinations}

Slit-lamp examinations with photographic documentation will be performed at baseline. The diagnosis will be confirmed and the grade of cataract determined by two independent ophthalmologists. The amplitude of pseudoaccommodation will be measured by the lens-loading method in an examination room under 350 lux illumination. Ocular higher order aberrations will be measured with a Pentacam (Oculus, Wetzler, Germany) and a KW-9000 aberrometer (Topcon, Tokyo, Japan). The measurements will be made through a $4 \mathrm{~mm}$ pupil diameter and repeated at least three times to acquire well focused and properly aligned images. The retinal and choroidal thickness will be measured by SD-OCT (RS-3000; NIDEK, Gamagori, Japan). The optical density of the macular pigment will be measured using a macular pigment screener (MPS III; Electron Technology, Cambridge, UK).

\section{Post-illumination pupil response}

Following pharmacological blockage of rod and cone cells in vitro, the melanopsin-associated ganglion cell response can be isolated as a slow, maintained depolarisation to light stimulation, which repolarises slowly after light offset. ${ }^{47}$ This PIPR is an index of the sensitivity of the melanopsin-containing ipRGC pathway. ${ }^{48}$ The PIPR will be measured using a pupillometer (RAPDx; Konan Medical Inc, Tokyo, Japan). The baseline pupil diameter will be measured after 10 min of dark adaptation. The diameters at baseline, the peak and during sustained pupillary constriction, will be analysed after $10 \mathrm{~s}$ of blue light $(440 \mathrm{~nm})$ and red light $(605 \mathrm{~nm})$ stimulation. Baseline pupil diameter is the average pupil diameter during a $7 \mathrm{~s}$ period before light onset. Sustained pupil diameter is the average from 10 to $40 \mathrm{~s}$ after light offset. The PIPR (mm), PIPR change (\%), net PIPR $(\mathrm{mm})$ and net PIPR change $(\%)$ will be calculated as follows. ${ }^{49}$

$$
\begin{aligned}
\operatorname{PIPR}(\mathrm{mm})= & \text { Baseline pupil diameter }(\mathrm{mm}) \\
& - \text { Sustained pupil diameter }(\mathrm{mm})
\end{aligned}
$$

PIPR change $(\%)=($ PIPR $/$ Baseline pupil diameter $) \times 100$

$$
\text { Net PIPR }(\mathrm{mm})=\text { Blue PIPR }- \text { Red PIPR }
$$

$$
\begin{aligned}
\text { Net PIPR change }(\%)= & \text { Blue PIPR change }(\%) \\
& - \text { Red PIPR change }(\%)
\end{aligned}
$$


Table 1 Schedule of participants visit and data collection

\begin{tabular}{|c|c|c|c|c|c|}
\hline & Enrolment & $\begin{array}{l}\text { Allocation/ } \\
\text { baseline }\end{array}$ & Intervention* & $\begin{array}{l}1 \text { year after } \\
\text { intervention }\end{array}$ & $\begin{array}{l}\text { Annual follow-up } \\
\text { from } 2 \text { to } 20 \text { years } \\
\text { after intervention }\end{array}$ \\
\hline \multicolumn{6}{|l|}{ Eligibility screen } \\
\hline Slit-lamp examination & $\checkmark$ & & & & \\
\hline Fundal examination & $\checkmark$ & & & & \\
\hline Intraocular pressure & $\checkmark$ & & & & \\
\hline \multicolumn{6}{|l|}{ Outcomes assessment } \\
\hline Mortality & & $\checkmark$ & & $\checkmark$ & $\checkmark$ \\
\hline Incidence of CVD, cancer & & $\checkmark$ & & $\checkmark$ & $\checkmark$ \\
\hline PSQI, ESS, GDS-15, NEI VFQ25 & & $\checkmark$ & & $\checkmark$ & $\checkmark$ \\
\hline Fundal examination & & $\checkmark$ & & $\checkmark$ & $\checkmark$ \\
\hline SD-OCT & & $\checkmark$ & & $\checkmark$ & $\checkmark$ \\
\hline Density of the macular pigment & & $\checkmark$ & & $\checkmark$ & $\checkmark$ \\
\hline Pseudoaccommodation aberration & & $\checkmark$ & & $\checkmark$ & \\
\hline Glucose, HbA1c, TG, LDL/HDL C & & $\checkmark$ & & $\checkmark$ & \\
\hline BMI, abdominal circumference & & $\checkmark$ & & $\checkmark$ & \\
\hline Actigraphy, wrist skin temperature & & $\checkmark$ & & $\checkmark$ & \\
\hline Urinary 6-sulfatoymelatonin & & $\checkmark$ & & $\checkmark$ & \\
\hline PIPR & & $\checkmark$ & & $\checkmark$ & \\
\hline
\end{tabular}

\section{Self-reported questionnaires}

Depressive symptoms will be assessed using the GDS-15, which is a self-administered questionnaire consisting of 15 items. $^{50}$ The validity of the questionnaire has been established previously. ${ }^{51}{ }^{52}$ Subjective sleep quality and daytime sleepiness will be assessed using the PSQI ${ }^{53}$ and the ESS, ${ }^{54}$ respectively. Chronotype and subjective visual function will be determined using the MorningnessEveningness Questionnaire, ${ }^{55}$ the Munich Chronotype Questionnaire $^{56}$ and the NEI VFQ25. ${ }^{57} 58$

\section{Analysis of venous blood sample and morning spot urine}

Overnight fasting venous blood samples and morning spot urine samples will be obtained at baseline and 1 year after surgery and will be analysed at a commercial laboratory (SRL Co, Inc, Tokyo, Japan) using standard clinical chemistry analysis to determine the concentrations of HbA1c, FPG, TG, LDL-C and HDL-C. The urinary aMT6-s concentration will be measured using an ELISA kit (RE54031; IBL International, Hamburg, Germany). Peak nocturnal plasma melatonin is significantly associated with aMT6-s in subsequent morning spot urine $(\mathrm{r}=0.69){ }^{59} 60$

\section{Actigraphic sleep and circadian activity rhythm}

Participants will wear an actigraph (ActiSleep-BT Monitor; ActiGraph Inc, Florida, USA) on the nondominant arm for 5 days including weekdays and a weekend, and will keep a sleep diary logging bedtime and rising time. Total sleep time, sleep efficiency, sleep-onset latency and wake after sleep onset will be calculated with ActiLife 6 (ActiGraph Inc). Indices of sleep quality using this device show moderate-to-high agreement with sleep parameters measured by polysomnography. ${ }^{61}$ Actigraphic data show the circadian physical activity rhythm. According to large-scale prospective cohort studies, decreased amplitude, later phase and decreased robustness of circadian activity rhythm analysed using sigmoidally transformed cosine curves ${ }^{62}$ show a significantly higher HR for incidence of cognitive disorders, cancer mortality and all-cause mortality. ${ }^{63-65}$

\section{Wrist skin temperature}

Wrist skin temperature reveals a mirror image of core body temperature, ${ }^{667}$ and an evening increase in wrist temperature is significantly correlated with the time of dim light melatonin onset in real-life situations $(\mathrm{r}=0.76) .{ }^{68}$ Wrist skin temperature will be measured on the inside of the wrist, near the radial artery of the nondominant arm at 3 min intervals using a temperature data logger (Thermochron iButton; Maxim/Dallas, Dallas, Texas, USA).

\section{Sample size calculation}

Of 2636 participants aged 60 years or older at enrolment in the Blue Mountains Eye Study, ${ }^{43}$ 27\% (n=713) died during 10 years of follow-up. To detect a $7.6 \%$ reduction in the risk of death over 10 years with a $95 \%$ two-sided $\alpha$ level of $5 \%$ with a power of $80 \%, 481$ participants in each group would be required. Assuming a dropout rate of $3 \%$, a total of 1000 participants would be needed. 


\section{Randomisation, masking}

Central randomisation by an independent allocator maintains allocation concealment. Random sequence was generated by computer. The results of allocation will be open to the care providers and the participants, but to outcome assessors.

\section{Statistical analyses}

Outcomes will be compared between the clear IOL group and the blue-blocking IOL group based on the intention-to-treat principle. For missing values due to loss to follow-up after baseline measurement, baseline data will be imputed using the last-observation-carriedforward method. For continuous variables with normal distributions, the mean and SD will be reported. For variables not distributed normally, the median and IQR will be reported. Means, medians and proportions will be compared using the $\mathrm{t}$ test, the Mann-Whitney $\mathrm{U}$ test and the $\chi^{2}$ test, respectively. Analysis of covariance will be used to estimate adjusted mean values and $95 \%$ CIs. The prevalence of the two groups will be tested using multivariate logistic regression analysis. The Kaplan-Meier plot, the log-rank test and the Cox proportional hazard model will be used to compare the mortalities and disease incidence rates between the two groups.

\section{Data monitoring}

The frequency of the adverse events will be analysed according to medical records. A data monitoring committee consisting of researchers and external specialists in internal medicine, public health and ophthalmology will annually report the number of participants, adverse events and results of the interim analysis. The committee will make the final decision to terminate the trial. Annual audit will be conducted by the Institutional Review Board of Nara Medical University, independent of investigators.

\section{Ethics and dissemination}

All modifications to the protocol will be reported to the UMIN-CTR and communicated to the public. An appropriately trained ophthalmologist will obtain informed consent. To promote data quality, double data entry will be conducted. To assure confidentiality, all paper-based personal information, and blood and urine samples, will be coded by identification number without personal information and stored at Nara Medical University School of Medicine in locked cabinets or locked freezers with limited access. Electronic data will be stored on a secure password-protected server during the study. The findings of this study will be communicated to healthcare professionals, participants and the public through peerreviewed publications, scientific conferences and the UMIN-CTR home page.

\section{DISCUSSION}

The present study (CLOCK-IOL colour study) will be conducted simultaneously with another randomised controlled trial (the CLOCK-IOL study), which will investigate the influence of cataract surgery on circadian rhythm by comparing patients who receive cataract surgery with a control group at 3 months after baseline. ${ }^{69}$

There are two main limitations to this protocol. First, there is a lack of information about light exposure at night (LAN). If the amount of LAN is balanced between two groups due to random allocation, blue-blocking IOL may reveal a beneficial effect by reducing harmful influence of LAN according to recent cross-sectional evidence. The higher level of self-reported LAN asked by questionnaire was significantly associated with higher prevalence of obesity among over 100000 women. $^{70}$ Furthermore, objectively measured LAN in the bedroom also showed significant associations with a prevalence of obesity, ${ }^{71}$ depression, ${ }^{72}$ insomnia, ${ }^{73}$ nocturnal hypertension $^{74}$ and atherosclerosis. ${ }^{75}$

Second, a seasonal effect may modify outcomes such as the prevalence of depression and circadian rhythm parameters, for instance, urinary aMT6-s, wrist skin temperature and the circadian rhythm of physical activity, because participants will be recruited throughout the year. Seasonal variables such as day length and outdoor temperature will be taken into account during data analysis.

\section{Twitter Follow Toyoaki Matsuura at @nekochan}

Contributors TN, KS and KO designed the study. TN and KS prepared the first draft of the manuscript. KM, MY, NM, YM, HT, MO, TH, SM, MK, TU and TM provided ophthalmic expertise for the protocol for this clinical trial. NT contributed to the engineering aspect of measuring outcomes. NK and NO are grant holders. All authors contributed to the refinement of the study protocol and approved the final manuscript.

Funding The present study is supported by a grant for collaboration study from Nara Medical University.

Competing interests None declared.

Ethics approval The study protocol was approved by the Institutional Review Board of Nara Medical University (number 13-032) and was registered in the University Hospital Medical Information Network Clinical Trials Registry (UMIN-CTR; trial ID: UMIN000014680) on 28 July 2014, before the enrolment of the participants.

Provenance and peer review Not commissioned; externally peer reviewed.

Open Access This is an Open Access article distributed in accordance with the Creative Commons Attribution Non Commercial (CC BY-NC 4.0) license, which permits others to distribute, remix, adapt, build upon this work noncommercially, and license their derivative works on different terms, provided the original work is properly cited and the use is non-commercial. See: http:// creativecommons.org/licenses/by-nc/4.0/

\section{REFERENCES}

1. Flo E, Pallesen S, Akerstedt T, et al. Shift-related sleep problems vary according to work schedule. Occup Environ Med 2013;70:238-45.

2. Feskanich D, Hankinson SE, Schernhammer ES. Nightshift work and fracture risk: the Nurses' Health Study. Osteoporos Int 2009;20:537-42. 
3. Karlsson B, Knutsson A, Lindahl B. Is there an association between shift work and having a metabolic syndrome? Results from a population based study of 27,485 people. Occup Environ Med 2001;58:747-52.

4. Pietroiusti A, Neri A, Somma G, et al. Incidence of metabolic syndrome among night-shift healthcare workers. Occup Environ Med 2010;67:54-7.

5. Morikawa $\mathrm{Y}$, Nakagawa $\mathrm{H}$, Miura $\mathrm{K}$, et al. Shift work and the risk of diabetes mellitus among Japanese male factory workers. Scand $J$ Work Environ Health 2005;31:179-83.

6. Kroenke $\mathrm{CH}$, Spiegelman D, Manson J, et al. Work characteristics and incidence of type 2 diabetes in women. Am J Epidemiol 2007; $165: 175-83$.

7. Bara AC, Arber S. Working shifts and mental health-findings from the British Household Panel Survey (1995-2005). Scand J Work Environ Health 2009;35:361-7.

8. Fujino $\mathrm{Y}$, Iso $\mathrm{H}$, Tamakoshi $\mathrm{A}$, et al. A prospective cohort study of shift work and risk of ischemic heart disease in Japanese male workers. Am J Epidemiol 2006;164:128-35.

9. Brown DL, Feskanich D, Sanchez BN, et al. Rotating night shift work and the risk of ischemic stroke. Am J Epidemiol 2009:169:1370-7.

10. Vyas MV, Garg AX, lansavichus AV, et al. Shift work and vascular events: systematic review and meta-analysis. BMJ 2012;345:e4800.

11. Schernhammer ES, Laden F, Speizer FE, et al. Rotating night shifts and risk of breast cancer in women participating in the nurses health study. J Natl Cancer Inst 2001;93:1563-8.

12. Schernhammer ES, Laden F, Speizer FE, et al. Night-shift work and risk of colorectal cancer in the nurses' health study. J Natl Cancer Inst 2003:95:825-8.

13. Kolstad HA. Nightshift work and risk of breast cancer and other cancers-a critical review of the epidemiologic evidence. Scand $J$ Work Environ Health 2008;34:5-22.

14. Poole EM, Schernhammer ES, Tworoger SS. Rotating night shift work and risk of ovarian cancer. Cancer Epidemiol Biomarkers Prev 2011;20:934-8.

15. Bhatti $P$, Mirick DK, Davis $S$. The impact of chronotype on melatonin levels among shift workers. Occup Environ Med 2014;71:195-200.

16. Scheer FA, Hilton MF, Mantzoros CS, et al. Adverse metabolic and cardiovascular consequences of circadian misalignment. Proc Natl Acad Sci USA 2009;106:4453-8.

17. Khalsa SB, Jewett ME, Cajochen $\mathrm{C}$, et al. A phase response curve to single bright light pulses in human subjects. J Physiol 2003;549 (Pt 3):945-52.

18. Pulivarthy SR, Tanaka N, Welsh DK, et al. Reciprocity between phase shifts and amplitude changes in the mammalian circadian clock. Proc Natl Acad Sci USA 2007;104:20356-61.

19. Hattar S, Liao HW, Takao M, et al. Melanopsin-containing retinal ganglion cells: architecture, projections, and intrinsic photosensitivity. Science 2002;295:1065-70.

20. Brainard GC, Hanifin JP, Greeson JM, et al. Action spectrum for melatonin regulation in humans: evidence for a novel circadian photoreceptor. J Neurosci 2001;21:6405-12.

21. Mariotti SP. Gloval data on visual impairments 2010. World Health Organization, 2014:1-14. http://www.who.int/blindness/ GLOBALDATAFINALforweb.pdf

22. Kessel L, Lundeman $\mathrm{JH}$, Herbst $\mathrm{K}$, et al. Age-related changes in the transmission properties of the human lens and their relevance to circadian entrainment. J Cataract Refract Surg 2010;36:308-12.

23. Danquah L, Kuper H, Eusebio C, et al. The long term impact of cataract surgery on quality of life, activities and poverty: results from a six year longitudinal study in Bangladesh and the Philippines. PLOS ONE 2014;9:e94140.

24. Asplund R, Ejdervik Lindblad B. The development of sleep in persons undergoing cataract surgery. Arch Gerontol Geriatr 2002;35:179-87.

25. Ayaki M, Muramatsu M, Negishi K, et al. Improvements in sleep quality and gait speed after cataract surgery. Rejuvenation Res 2013;16:35-42.

26. Wei $X$, She $C$, Chen $D$, et al. Blue-light-blocking intraocular lens implantation improves the sleep quality of cataract patients. J Clin Sleep Med 2013;9:741-5.

27. Alexander I, Cuthbertson FM, Ratnarajan G, et al. Impact of cataract surgery on sleep in patients receiving either ultraviolet-blocking or blue-filtering intraocular lens implants. Invest Ophthalmol Vis Sci 2014:55:4999-5004.

28. Gray CS, Karimova G, Hildreth AJ, et al. Recovery of visual and functional disability following cataract surgery in older people: Sunderland Cataract Study. J Cataract Refract Surg 2006;32:60-6.

29. Ishii K, Kabata T, Oshika T. The impact of cataract surgery on cognitive impairment and depressive mental status in elderly patients. Am J Ophthalmol 2008;146:404-9.
30. Fraser ML, Meuleners LB, Lee AH, et al. Vision, quality of life and depressive symptoms after first eye cataract surgery. Psychogeriatrics 2013;13:237-43.

31. Meuleners LB, Hendrie D, Fraser ML, et al. The impact of first eye cataract surgery on mental health contacts for depression and/or anxiety: a population-based study using linked data. Acta Ophthalmol 2013;91:e445-9.

32. Golden RN, Gaynes BN, Ekstrom RD, et al. The efficacy of light therapy in the treatment of mood disorders: a review and meta-analysis of the evidence. Am J Psychiatry 2005;162:656-62.

33. Lieverse R, Van Someren EJ, Nielen MM, et al. Bright light treatment in elderly patients with nonseasonal major depressive disorder: a randomized placebo-controlled trial. Arch Gen Psychiatry 2011;68:61-70.

34. Tanito M, Okuno T, Ishiba $\mathrm{Y}$, et al. Transmission spectrums and retinal blue-light irradiance values of untinted and yellow-tinted intraocular lenses. J Cataract Refract Surg 2010;36:299-307.

35. Mainster MA, Turner PL. Blue-blocking IOLs decrease photoreception without providing significant photoprotection. Surv Ophthalmol 2010;55:272-89.

36. Turner PL, Van Someren EJ, Mainster MA. The role of environmental light in sleep and health: effects of ocular aging and cataract surgery. Sleep Med Rev 2010;14:269-80.

37. Ham WT Jr, Ruffolo JJ Jr, Mueller HA, et al. Histologic analysis of photochemical lesions produced in rhesus retina by short-wavelength light. Invest Ophthalmol Vis Sci 1978;17:1029-35.

38. Sparrow JR, Nakanishi K, Parish CA. The lipofuscin fluorophore A2E mediates blue light-induced damage to retinal pigmented epithelial cells. Invest Ophthalmol Vis Sci 2000;41:1981-9.

39. Algvere PV, Marshall J, Seregard S. Age-related maculopathy and the impact of blue light hazard. Acta Ophthalmol Scand 2006;84:4-15.

40. Klein R, Klein BE, Jensen SC, et al. The relationship of ocular factors to the incidence and progression of age-related maculopathy. Arch Ophthalmol 1998:116:506-13.

41. Klein R, Klein BE, Wong TY, et al. The association of cataract and cataract surgery with the long-term incidence of age-related maculopathy: the Beaver Dam eye study. Arch Ophthalmol 2002;120:1551-8.

42. Wang JJ, Klein R, Smith W, et al. Cataract surgery and the 5-year incidence of late-stage age-related maculopathy: pooled findings from the Beaver Dam and Blue Mountains eye studies. Ophthalmology 2003;110:1960-7.

43. Cugati S, Mitchell $P$, Rochtchina E, et al. Cataract surgery and the 10-year incidence of age-related maculopathy: the Blue Mountains Eye Study. Ophthalmology 2006;113:2020-5.

44. Sparrow JR, Miller AS, Zhou J. Blue light-absorbing intraocular lens and retinal pigment epithelium protection in vitro. $J$ Cataract Refract Surg 2004;30:873-8.

45. Chylack LT Jr, Wolfe JK, Singer DM, et al. The Lens Opacities Classification System III. The Longitudinal Study of Cataract Study Group. Arch Ophthalmol 1993;111:831-6.

46. Sanders DR, Retzlaff J, Kraff MC. Comparison of empirically derived and theoretical aphakic refraction formulas. Arch Ophthalmol 1983;101:965-7.

47. Dacey DM, Liao HW, Peterson BB, et al. Melanopsin-expressing ganglion cells in primate retina signal colour and irradiance and project to the LGN. Nature 2005;433:749-54.

48. Gamlin PD, McDougal DH, Pokorny J, et al. Human and macaque pupil responses driven by melanopsin-containing retinal ganglion cells. Vision Res 2007;47:946-54.

49. Kankipati L, Girkin CA, Gamlin PD. Post-illumination pupil response in subjects without ocular disease. Invest Ophthalmol Vis Sci 2010;51:2764-9.

50. Burke WJ, Roccaforte WH, Wengel SP. The short form of the Geriatric Depression Scale: a comparison with the 30-item form. $J$ Geriatr Psychiatry Neurol 1991;4:173-8.

51. Wancata J, Alexandrowicz R, Marquart B, et al. The criterion validity of the Geriatric Depression Scale: a systematic review. Acta Psychiatr Scand 2006;114:398-410.

52. Almeida OP, Almeida SA. Short versions of the geriatric depression scale: a study of their validity for the diagnosis of a major depressive episode according to ICD-10 and DSM-IV. Int J Geriatr Psychiatry 1999; 14:858-65.

53. Buysee DJ, Reynolds CF III, Monk TH, et al. The Pittsburgeh Sleep Quality Index: a new instrument for psychiatric practice and research. Psychiatry Res 1988;28:193-213.

54. Johns MW. Reliability and factor analysis of the Epworth Sleepiness Scale. Sleep 1992;15:376-81. 
55. Horne JA, Ostberg O. A self-assessment questionnaire to determine morningness-eveningness in human circadian rhythms. Int $J$ Chronobiol 1976;4:97-110.

56. Kitamura S, Hida A, Aritake S, et al. Validity of the Japanese version of the Munich ChronoType Questionnaire. Chronobiol Int 2014;31:845-50.

57. Mangione CM, Lee PP, Gutierrez PR, et al. Development of the 25-item National Eye Institute Visual Function Questionnaire. Arch Ophthalmol 2001;119:1050-8.

58. Suzukamo Y, Oshika T, Yuzawa M, et al. Psychometric properties of the 25-item National Eye Institute Visual Function Questionnaire (NEI VFQ-25), Japanese version. Health Qual Life Outcomes 2005:3:65.

59. Graham C, Cook M, Kavet R, et al. Prediction of nocturnal plasma melatonin form morning urinary measures. J Pineal Res 1998;24:230-8.

60. Schernhammer ES, Rosner B, Wilett W, et al. Epidemiology of urinary melatonin in women and its relation to other hormones and night work. Cancer Epidemiol Biomarkers Prev 2004;13:936-43.

61. Cellini N, Burman M, McDevitt E, et al. Direct Comparison of two actigraphy devices with polysomnographically recorded naps in healthy young adults. Chronobiology Int 2013;30:691-8.

62. Marler MR, Gehrman P, Martin JL, et al. The sigmoidally transformed cosine curve: a mathematical model for circadian rhythms with symmetric non-sinusoidal shapes. Stat Med 2006;25:3893-904.

63. Tranah GJ, Blackwell T, Ancoli-Israel S, et al. Circadian activity rhythms and mortality: the study of osteoporotic fractures. J Am Geriatr Soc 2010;58:282-91.

64. Paudel ML, Taylor BC, Ancoli-Israel S, et al. Rest/activity rhythms and cardiovascular disease in older men. Chronobiol Int 2011;28:258-66.

65. Tranah GJ, Blackwell T, Stone KL, et al. Circadian activity rhythms and risk of incident dementia and mild cognitive impairment in older women. Ann Neurol 2011;70:722-32.
66. Sarabia JA, Rol MA, Mendiola P, et al. Circadian rhythm of wrist temperature in normal-living subjects a candidate of new index of the circadian system. Physiol Behav 2008;95:570-80.

67. Krauchi K, Wirz-Justice A. Circadian rhythm of heat production, heart rate, and skin and core temperature under unmasking conditions in men. Am J Physiol 1994;267(3 Pt 2):R819-29.

68. Bonmati-Carrion MA, Middleton B, Revell V, et al. Circadian phase assessment by ambulatory monitoring in humans: correlation with dim light melatonin onset. Chronobiol Int 2014;31:37-51.

69. Saeki K, Obayashi K, Nishi T, et al. Short-term influence of cataract surgery on circadian biological rhythm and related health outcomes (CLOCK-IOL trial): study protocol for a randomized controlled trial. Trials 2014;15:514.

70. McFadden E, Jones ME, Schoemaker MJ, et al. The relationship between obesity and exposure to light at night: cross-sectional analyses of over 100,000 women in the Breakthrough Generations Study. Am J Epidemiol 2014;180:245-50.

71. Obayashi K, Saeki K, Iwamoto J, et al. Exposure to light at night, nocturnal urinary melatonin excretion, and obesity/dyslipidemia in the elderly: a cross-sectional analysis of the HEIJO-KYO study. $J$ Clin Endocrinol Metab 2013;98:337-44.

72. Obayashi K, Saeki K, Iwamoto J, et al. Exposure to light at night and risk of depression in the elderly. J Affect Disord 2013;151:331-6.

73. Obayashi K, Saeki K, Kurumatani N. Association between light exposure at night and insomnia in the general elderly population: the HEIJO-KYO cohort. Chronobiol Int 2014;31:976-82.

74. Obayashi K, Saeki K, Iwamoto J, et al. Association between light exposure at night and nighttime blood pressure in the elderly independent of nocturnal urinary melatonin excretion. Chronobiol Int 2014;31:779-86.

75. Obayashi K, Saeki K, Kurumatani N. Light exposure at night is associated with subclinical carotid atherosclerosis in the general elderly population: the HEIJO-KYO cohort. Chronobiol Int 2015;32:310-17. 